\title{
A Hybrid Wavelet Fuzzy Neural Network and Switching Particle Swarm Optimization Algorithm for AC Servo System
}

\author{
Run-min Hou, Yuan-long Hou, Chao Wang, Qiang Gao, and Hao Sun \\ Department of Mechanical Engineering, Nanjing University of Science and Technology, Nanjing 210094, China \\ Correspondence should be addressed to Run-min Hou; 187189579@qq.com
}

Received 6 May 2016; Revised 4 October 2016; Accepted 3 November 2016

Academic Editor: Andrea L. Facci

Copyright (C) 2016 Run-min Hou et al. This is an open access article distributed under the Creative Commons Attribution License, which permits unrestricted use, distribution, and reproduction in any medium, provided the original work is properly cited.

\begin{abstract}
A hybrid computational intelligent approach which combines wavelet fuzzy neural network (WFNN) with switching particle swarm optimization (SPSO) algorithm is proposed to control the nonlinearity, wide variation in loads, time variation, and uncertain disturbance of the high-power AC servo system. The WFNN method integrated wavelet transforms with fuzzy rules and is proposed to achieve precise positioning control of the AC servo system. As the WFNN controller, the back-propagation method is used for the online learning algorithm. Moreover, the SPSO is proposed to adapt the learning rates of the WFNN online, where the velocity updating equation is according to a Markov chain, which makes it easy to jump the local minimum, and acceleration coefficients are dependent on mode switching. Furthermore, the stability of the closed loop system is guaranteed by using the Lyapunov method. The results of the simulation and the prototype test prove that the proposed approach can improve the steady-state performance and possess strong robustness to both parameter perturbation and load disturbance.
\end{abstract}

\section{Introduction}

In the recent years, with the advancement of technology, $\mathrm{AC}$ servo system has been widely used. As a servo drive system, it needs not only a good steady-state performance, but also a high dynamic performance. As a controlled object, the dynamic mathematical model of a high-power AC motor is a complex system, which is characterized by heavy varying load, slow time variation, nonlinearity, and uncertain disturbance. Traditional control algorithm adopts PID control, easily influenced by the model of nonlinear characteristics and the parameters such as the uncertainty of dynamic response and the unbalance of antidisturbance ability, which may deteriorate the system control performance. Thus, the practical intelligent control strategy has become a focus in the field of servo system control [1].

As neural networks may approach any nonlinear function, they have already been widely utilized in the modeling of nonlinear systems and have advantages of easy realization and learning capability $[2,3]$. Some scholars $[4,5]$ construct the motor servo system model by using neural network and obtain the conclusion that the application accuracy of the neural network in the identification of nonlinear systems is higher than that of the linear system. However, in the neural network, the sigmoid function is used as the activation function of BP neural network, which leads to the result that the BP neural network is easy to get into local minimum, slow convergence speed, and difficult to understand the mapping rules which make it impossible to be used in realtime tasks. Fuzzy logic uses human-like reasoning and expert knowledge to model complex and uncertain systems $[6,7]$. Some researchers have proposed various structures for modeling and controlling of nonlinear systems $[8,9]$. Reference [10] uses fuzzy control and has the ability to deal with the uncertainty of self-learning ability of the neural network by combining fuzzy neural network and its application in the controller of a servo motor, which effectively improves the robustness of the system and does not require accurate mathematical model of the controlled object. The main features are as follows: (1) the use of fuzzy neural network tuning fuzzy membership functions; (2) the inference rules of logic systems; (3) the use of construction in the form of inference rules propagation network structure to realize the benefits of functional complementarily. However, in the common conditions, these parameters learning algorithm require presetting fuzzy system topology. 
As an alternative, wavelet neural network is a feedforward network based on wavelet analysis, effectively combining the structural model of neural network, and the determination of the entire network structure has a reliable theoretical basis and thereby avoids the blindness of the structural design. Many researchers have proposed wavelet fuzzy neural network (WFNN) combining wavelet theory with fuzzy neural network (FNN) [11-14]. In WFNN, each fuzzy rule corresponds to sub-WNN, and the approximation accuracy of the WFNN can be improved greatly by learning the setting parameters of wavelet and fuzzy [15]. Although the WNN has been successfully applied in nonlinear system, some challenging issues still exist, such as how to optimize the structure of WNN.

Particle swarm optimization (PSO) algorithm is a global optimization algorithm, through collaboration and competition between individuals to find the optimal solution, and particle swarm optimization search process is started from the entire group, with the implicit parallel search features to improve the performance of the algorithm [16]. However, the PSO algorithm has some disadvantages such as easily falling into local minima and slow convergence speed. In order to overcome these drawbacks, a new SPSO algorithm is proposed to train the FWNN in this paper. The SPSO algorithm $[17,18]$ has been using velocity updating equation with Markovian switching parameters to overcome the contradiction between the local search and the global search. The proposed SPSO algorithm can not only avoid the local search stagnating in a local area but also lead the swarm to move to a more potential area quickly. Therefore, the SPSO algorithm can greatly improve the ability of WNN and global search.

In this study, in order to achieve control over the highpower AC servo system, a WFNN is applied to build the intelligent model and control for this system, which is trained by means of learning rate with using SPSO algorithm. The convergence rate is greatly accelerated, and the local optimum is avoided. Finally, simulation results illustrating the validity and advantages of the proposed WFNN for the AC servo control system are discussed.

This paper is organized as follows. In Section 2, the servo system is being analyzed. The structure of the fuzzy wavelet neural network (FWNN) and the structure learning and parameter learning algorithms are introduced in Sections 3 and 4 . In addition, the design procedures, adaptive learning algorithms, and the stability analysis of the proposed FWNN controller are also described in detail in Section 4. Simulation results are discussed in Sections 5 and 6; last part gives the conclusion of this paper.

\section{Modeling AC Servo System}

The control structure chart of an AC servo system is presented in Figure 1. Due to the nonlinearity of the motor itself, the nonlinear phase comparison brought by the system load changes is very small, so the derivation of the model makes some assumptions [19]: (a) no saturation effect; (b) motor evenly distributed air gap and magnetic induction
EMF sinusoidal shape; (c) excluding the hysteresis and eddy current loss; (d) no rotor excitation winding.

In Figure 1, the dotted line is the control schematic of an AC speed control system; $\theta_{d}$ is the target position; $\theta$ is the actual target position, respectively. In addition, $U$ is the control voltage; $K_{\alpha}$ is the amplifier gain; $R$ is the loop resistance of motor armature; $L$ is the loop inductance of motor armature; $K_{d}$ is the motor torque coefficient; $T_{d}$ is the motor electromagnetic torque; $E_{e}$ is the counter-electromotive force of motor armature; $C_{e}$ is the counter-electromotive force coefficient of motor; $T_{L}$ is the load disturbance torque; $T_{f}$ is the friction torque disturbance; $J$ is the total inertia moment converted to the rotor; $B$ is the viscous friction coefficient; $\omega_{d}$ is the angular velocity of motor; $i$ is the reduction ratio.

Generally, the current time constant in the motor is much smaller than the mechanical time constant, so the delay time of current response can be ignored [20,21]

$$
\frac{1}{L s+R}=\frac{1}{R} \frac{1}{L s / R+1} \approx \frac{1}{R} \text {. }
$$

The electromagnetic torque of motor is

$$
T_{d}=-\frac{K_{d} C_{e}}{R} \omega_{d}+\frac{K_{d} K_{\alpha}}{R} U .
$$

The torque balance equation can be shown as

$$
T_{d}-T_{L}-T_{f}=J i \ddot{\theta}+B i \dot{\theta} .
$$

Putting (2) into (3), (3) can be rewritten as

$$
J i \ddot{\theta}+B i \dot{\theta}=-\frac{K_{d} C_{e}}{R} \omega_{d}+\frac{K_{d} K_{\alpha}}{R} U-T_{L}-T_{f} .
$$

Multiplying both sides of (4) by $1 / i$, it can be shown as

$$
\ddot{\theta}=-\left(\frac{B}{J}+\frac{K_{d} C_{e}}{J R}\right) \dot{\theta}+\frac{K_{d} K_{\alpha}}{i J R} U-\frac{T_{L}+T_{f}}{i J} .
$$

Set state variable $x=\left[\begin{array}{ll}x_{1} & x_{2}\end{array}\right]^{T}, x_{1}=\theta$ and $x_{2}=\dot{\theta}$, and then the state space equation of the speed control system is

$$
\begin{aligned}
& \dot{x}_{1}=x_{2}, \\
& \dot{x}_{2}=f(x)+g u(t)+d(t),
\end{aligned}
$$

where $f(x)=-\left(B / J+K_{d} C_{e} / J R\right) x_{2} ; g=K_{d} K_{\alpha} / i J R ; d(t)=$ $-\left(T_{L}+T_{f}\right) / i J$; and $|d(t)| \leq C, C$ is a constant.

The system rotational inertia $J$, the viscous friction coefficient $B$, the load disturbance torque $T_{L}$, and the friction torque disturbance $T_{f}$ change when the work condition changes. That is to say, $f(x), g$, and $d(t)$ are all nonlinear functions.

\section{Wavelet Fuzzy Neural Network Controller}

Fuzzy wavelet neural network is made up of multiresolution of wavelet variation and traditional TSK fuzzy system. In fuzzy wavelet network, each fuzzy rule corresponds to given 


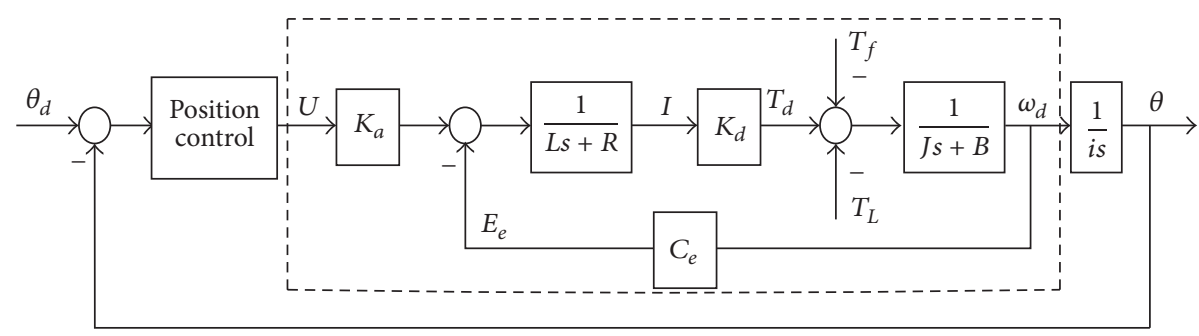

Figure 1: The diagram of the AC servo system.

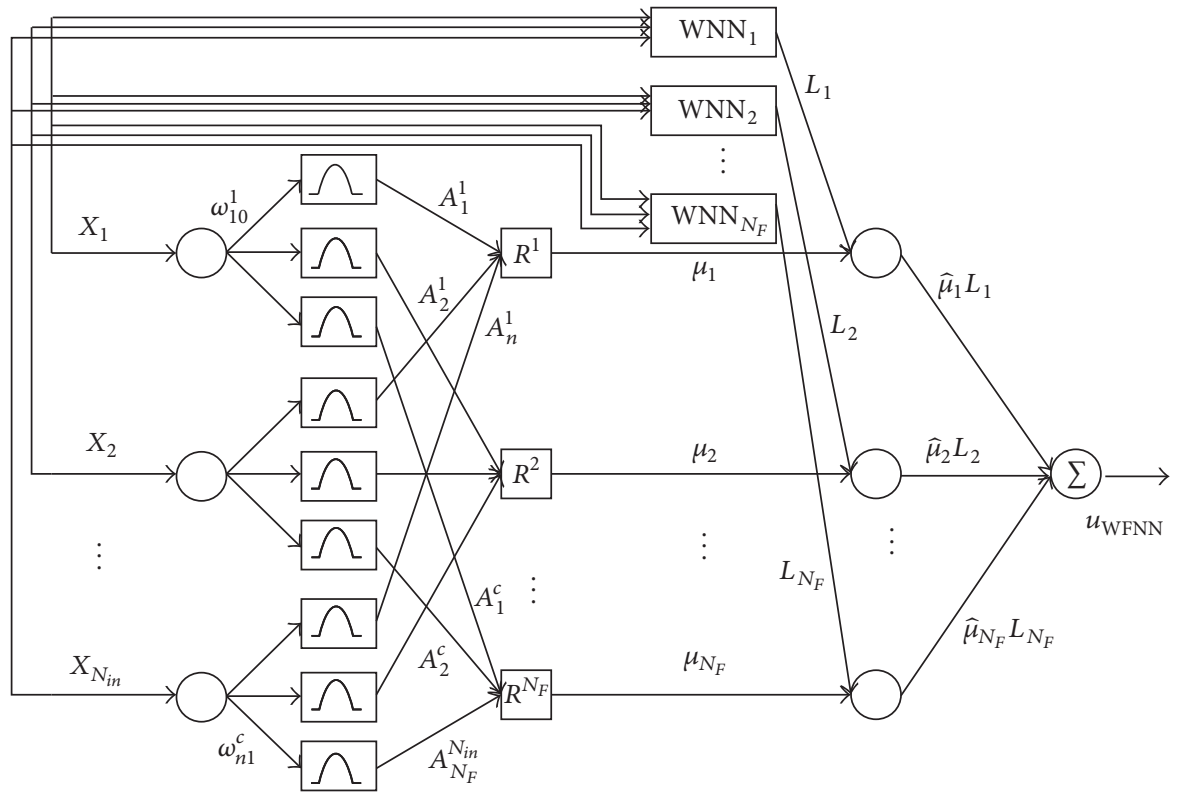

FIGURE 2: Structure of proposed WFNN.

wavelet scale values of wavelet neural network [11]. The structures integrate into sub-WNN and are shown in Figure 2.

The $n$th fuzzy rule of the proposed WFNN can be presented as follows.

$$
\begin{aligned}
& R_{n}: \text { if } x_{1} \text { is } A_{1 n}, x_{2} \text { is } A_{2 n}, \ldots \text {, and } x_{m} \text { is } A_{m n} \text {, then } \\
& y_{n}=L_{n}=\sum_{j=1}^{N_{w, n}} \omega_{j, n} \psi_{j, n}(x),
\end{aligned}
$$

where $R_{n}$ is the fuzzy rule $\left(1 \leq n \leq N_{F}\right) ; A_{m n}$ is the membership function for the fuzzy set of Gaussian function, which can be expressed as follows:

$$
\mu_{A_{m n}}\left(x_{m}\right)=\exp ^{\left(-\left(x_{m}-c_{m n}\right)^{2} / \sigma_{m n}^{2}\right)},
$$

where $x_{m}$ is the input of $m=1, \ldots, N_{i n} ; n=1, \ldots, N_{F}$. And a membership function is defined by a center $c_{m n}$ and a width $\sigma_{m n}$.

For the $N_{F}$ consequent part, the output $L_{N_{F}}$ of sub-WNN is shown as

$$
L=\sum_{j=1}^{N_{F}} \omega_{j} \psi_{j}(x),
$$

where $\omega_{j}$ are the wavelet weights and $\psi_{j}(x)$ are multidimensional wavelets which are generated from the mother wavelet function:

$$
\phi_{j k}\left(z_{j k}\right)=\phi_{j k} \frac{u_{j k}-m_{j k}}{d_{j k}},
$$

where $z_{j k}=\left(u_{j k}-m_{j k}\right) / d_{j k}, u_{j k}$ represents the output, $m_{j k}$ represents the shift factor, $d_{j k}$ represents the spreading factor, and $j, k$, respectively, represent the $j$ th wavelet of the $k$ th input item.

Using product rule and defuzzification, the output of the whole proposed WFNN structure is given by

$$
u_{\mathrm{WFNN}}(k)=\sum_{n=1}^{N_{F}} \widehat{\mu}_{n}(x) L_{n},
$$

where $\widehat{\mu}_{n}(x)=\mu_{n}(x) / \sum_{n=1}^{N_{F}} \mu_{n}(x), L_{n}=\sum_{j=1}^{N_{W(n)}} \omega_{j} \psi_{j}$, and $\mu_{n}(x)=\prod_{m} \mu_{A_{m n}}\left(x_{m}\right)$.

In order to make the system convergence faster and avoid the local optimum problem, the BP algorithm is used for realtime training. The energy function $J$ is defined as

$$
J(k)=\frac{1}{2}\left[\left(y_{d}(k)-y(k)\right)^{2}\right]=\frac{1}{2} e(k) .
$$


In order to facilitate training WNN controller parameters, set vector $\mathbf{P}_{n}$ as

$$
\begin{aligned}
\mathbf{P}_{n} & =\left[P_{n}^{1}, \ldots, P_{n}^{2}, \ldots, P_{n}^{l}\right] \\
& =\left[\omega_{j,(n)}, m_{j k,(n)}, d_{j k,(n)}, c_{j k,(n)}, \sigma_{j k,(n)}\right],
\end{aligned}
$$

where $n=1, \ldots, N_{F}, l=1, \ldots, 5, j=1, \ldots, N_{w, n}$, and $k=$ $1, \ldots, N_{\text {in }}$.

Use an adaptive learning rate BP algorithm for any parameter vector for real-time training, which can be expressed as

$$
P_{n}^{l}(k+1)=P_{n}^{l}(k)+\gamma_{c}^{l}\left(-\frac{\partial J_{1}(k)}{\partial P_{n}^{l}(k)}\right),
$$

where $\gamma_{c}^{l}$ is learning rate of WFNN controller parameters and is expressed as

$$
\gamma^{l}=\left[\gamma^{1}, \ldots, \gamma^{2}, \ldots, \gamma^{l}\right]=\left[\gamma^{\omega}, \gamma^{m}, \gamma^{d}, \gamma^{c}, \gamma^{\sigma}\right]
$$

For any system parameter vector $P_{n}^{l}$ of gradient performance is expressed as

$$
\frac{\partial J_{1}(k)}{\partial P_{n}^{l}(k)}=y(k)\left(\frac{\partial y(k)}{\partial u(k)}\right) \times \frac{\mu_{n}(x)}{\sum_{h=1}^{N_{F}} \mu(x)} \frac{\partial L_{n}}{\partial P_{n}^{l}(k)},
$$

where $\partial L_{n} / \partial P_{n}^{l}(k)$ can be shown as [22]

$$
\begin{aligned}
\frac{\partial L_{n}}{\partial P_{n}^{1}(k)} & =\frac{\partial L_{n}}{\partial \omega_{j, n}(k)}=\psi_{j, n}, \\
\frac{\partial L_{n}}{\partial P_{n}^{2}(k)} & =\frac{\partial L_{n}}{\partial m_{j k,(n)}(k)} \\
& =\omega_{j, n} \psi_{j, n}\left(\frac{-1}{d_{j k,(n)}}\right) \times\left(\frac{1}{z_{j k,(n)}}-z_{j k,(n)}\right), \\
\frac{\partial L_{n}}{\partial P_{n}^{3}(k)} & =\frac{\partial L_{n}}{\partial d_{j k,(n)}(k)}=z_{j k,(n)} \frac{\partial L_{n}}{\partial m_{j k,(n)}(k)}, \\
\frac{\partial L_{n}}{\partial P_{n}^{4}(k)} & =\frac{\partial L_{n}}{\partial c_{j k,(n)}(k)}=\frac{\left(x_{m}-c_{m n}\right)}{\sigma_{m n}^{2}}, \\
\frac{\partial L_{n}}{\partial P_{n}^{5}(k)} & =\frac{\partial L_{n}}{\partial \sigma_{j k,(n)}(k)}=\frac{\left(x_{m}-\sigma_{m n}^{2}\right)}{\sigma_{m n}^{3}} .
\end{aligned}
$$

Since the accuracy requirements within the scope of the error between the network identification output and the actual output of the controlled object in the offline training and learning, it can be considered that the actual output is replaced approximately by the identification output, which may not greatly affect the control performance. Then the controlled object gradient information $\partial y(k) / \partial u(k)$ of the learning algorithm of the WFNN controller was replaced by using the training algorithm of WFNN identification output [23].

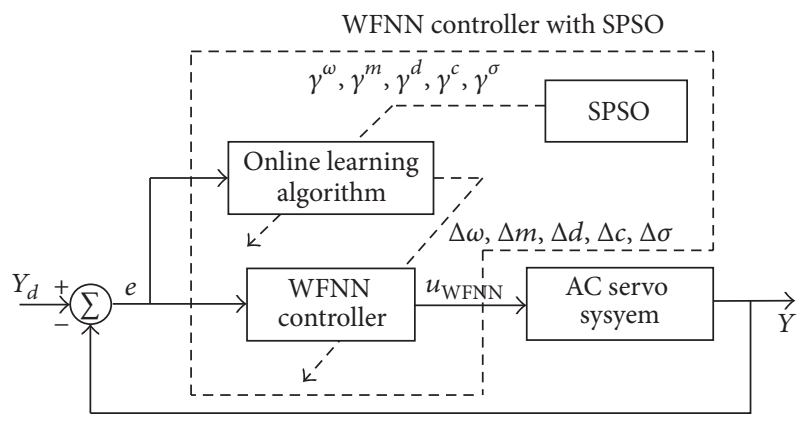

FIGURE 3: AC servo system using the proposed WFNN with SPSO.

\section{Training Algorithm and Stability Analysis}

4.1. A Novel Switching PSO. To improve the online learning capability of the proposed WFNN, the SPSO algorithm is adopted in this section to adapt the learning rates. The configuration of the proposed WFNN controller with SPSO is shown in Figure 3. A velocity updated equation with Markovian switching parameters is proposed to overcome the contradiction between the local search and global search. Markov chain is a very useful and important tool in control system [24]. At each step, the Markov process changes its state according to a certain probability distribution. The distribution properties can be described by evolutionary factor. This method can show the distance between the global best particle and other particles in the swarm. And it can be shown as follows:

$$
D=\frac{1}{S} \sum_{i=1}^{S} \sqrt{\sum_{d=1}^{D}\left(x_{i}^{d}-\bar{x}_{d}\right)^{2}}
$$

where $S$ and $D$ are the populations size and the dimensions, respectively. $x_{i}^{d}$ is the $d$ th value of the $i$ th particle, and $\bar{x}_{d}$ is the $d$ th value of the average point $\bar{x}$ in the whole swarm that can be shown as

$$
\bar{x}_{d}=\frac{1}{S} \sum_{i=1}^{S} x_{i d} .
$$

Due to the disadvantages of the classification PSO method (e.g., the swarm will be stagnated in local optimum if the current global best particle is a local optimum away from swarm), this will be reducing the convergence speed. Thus, a velocity updating equation with Markovian switching parameters to overcome this shortcoming and improve the search abilities is introduced in this section. The velocity and position updating equations with Markovian switching parameters are shown in the following equations:

$$
\begin{aligned}
v_{i}(k+1)= & w(k) v_{i}(k)+c_{1}(\xi(k)) r_{1}\left(p_{i}(k)-x_{i}(k)\right) \\
& +c_{2}(\xi(k)) r_{2}\left(p_{g}(k)-x_{i}(k)\right), \\
x_{i}(k+1)= & x_{i}(k)+v_{i}(k+1),
\end{aligned}
$$

where $c_{1}(\xi(k))$ and $c_{2}(\xi(k))$ are the acceleration coefficients. All of them are mode-dependent on a Markov chain. Let $\xi(k)$ 
be a Markov chain taking values in the finite state space $S=$ $\{1,2, \ldots, N\}$ with probability transition matrix $\Pi=\left(\pi_{i j}\right)_{N \times N}$ shown as

$$
\begin{aligned}
& P=\{\xi(k+1)=j \mid \xi(k)=i\}=\pi_{i j}, \\
& i, j=1,2, \ldots, N \text {, }
\end{aligned}
$$

where $\pi_{i j} \geq 0$ is the transition rate from $i$ to $j$ and $\sum_{j=1}^{N} \pi_{i j}=1$; $\xi(k)=1, \xi(k)=2, \xi(k)=3$, and $\xi(k)=4$ represent the states of convergence, exploration, exploitation, and jumping-out, respectively. The equations of classification and probability transition matrix are shown as follows [17]:

$$
\begin{aligned}
\xi(k) & = \begin{cases}1, & 0 \leq E_{f} \leq 0.25, \\
2, & 0.25 \leq E_{f} \leq 0.5, \\
3, & 0.5 \leq E_{f} \leq 0.75, \\
4, & 0.74 \leq E_{f} \leq 1,\end{cases} \\
\Pi= & \left(\begin{array}{cccc}
\eta & 1-\eta & 0 & 0 \\
\frac{1-\eta}{2} & \eta & \frac{1-\eta}{2} & 0 \\
0 & \frac{1-\eta}{2} & \eta & \frac{1-\eta}{2} \\
0 & 0 & 1-\eta & \eta
\end{array}\right),
\end{aligned}
$$

where $\eta$ is equal to 0.9 in the evolutionary process for keeping the classification accuracy as well as the search diversity; $E_{f}$ is evolution factor which is written as

$$
E_{f}=\frac{d_{g}-d_{\min }}{d_{\max }-d_{\min }}
$$

where $d_{g}$ represents the globally best particle in $D ; d_{\min }$ and $d_{\max }$ show the maximum and minimum distance in $D$, respectively.

The inertia weight $w$ is employed to balance the global and local search abilities. Thus, the inertia weight $w$ is defined as

$$
w\left(E_{f}\right)=0.5 E_{f}+0.4 \quad w \in[0.4,0.9], \forall E_{f}[0,1] .
$$

The inertia weight $w$ is monotonic with $E_{f}$ which makes $w$ adaptive to the search environment. Thus, the inertia weight is chosen as 0.9 in this paper. The flow chart of using the SPSO algorithm to optimize the parameters of WFNN is shown in Figure 4.

4.2. A Novel Stability Analysis. The Lyapunov function was used to judge the stability of the system. Define Lyapunov function:

$$
V(k)=\frac{1}{2} e(k),
$$

where $e(k)=\left(y_{d}(k)-y(k)\right)$.

$$
\Delta V(k)=\frac{1}{2}\left(e^{2}(k+1)-e^{2}(k)\right),
$$

where $e(k+1)=e(k)+\Delta e(k) . \Delta e(k)$ can be expanded by using Taylor formula:

$$
\Delta e(k)=\sum_{n=1}^{N_{F}}\left\{\left[\frac{\partial e(k)}{\partial P_{n}^{l}(k)}\right]^{T} \Delta P_{n}^{l}(k)\right\},
$$

where

$$
\begin{aligned}
& \frac{\partial e(k)}{\partial P_{n}^{l}(k)}=-A \frac{\mu_{n}(x)}{\sum_{h=1}^{N_{F}} \mu_{h}(x)} \frac{\partial L_{n}}{\partial P_{n}^{l}(k)}, \\
& \Delta P_{n}^{l}(k)=\gamma^{l} A e(k) \times \frac{\mu_{n}(x)}{\sum_{h=1}^{N_{F}} \mu_{h}(x)} \frac{\partial L_{n}}{\partial P_{n}^{l}(k)},
\end{aligned}
$$

where $A=\partial y(k) / \partial u(k)$.

$\Delta e(k)$ can be written by (29) and (28) as

$\Delta e(k)$

$$
=\gamma^{l} A^{2} e_{1}(k) \frac{1}{\left(\sum_{h=1}^{N_{F}} \mu_{h}(x)\right)^{2}} \sum_{n=1}^{N_{F}} \mu_{n}^{2}(x)\left\|\frac{\partial L_{n}}{\partial P_{n}^{l}(k)}\right\|^{2} .
$$

Putting (30) into (27), $\Delta V(k)$ can be rewritten as

$$
\begin{aligned}
\Delta V(k)= & -\frac{1}{\left(\sum_{h=1}^{N_{F}} \mu_{h}(x)\right)^{2}} \\
& \times \sum_{n=1}^{N_{F}}\left(\mu_{n}^{2}(x)\left\|\frac{\partial L_{n}}{\partial P_{n}^{l}(k)}\right\|^{2}\right) A e_{1}(k) \lambda,
\end{aligned}
$$

where

$$
\begin{aligned}
\lambda= & \gamma^{l}\left[1-\frac{1}{2} \frac{\gamma^{l}}{\left(\sum_{h=1}^{N_{F}} \mu_{h}(x)\right)^{2}}\right. \\
& \left.\times \sum_{n=1}^{N_{F}}\left(\mu_{n}^{2}(x)\left\|\frac{\partial L_{n}}{\partial P_{n}^{l}(k)}\right\|^{2}\right)\right] .
\end{aligned}
$$

When $\mu_{n}(x) \leq 1$, (32) can be rewritten as

$$
\begin{aligned}
\lambda \geq & \gamma^{l} \cdot\left[1-\frac{1}{2} \frac{\gamma^{l}}{\left(\sum_{h=1}^{N_{F}} \mu_{h}(x)\right)^{2}}\right. \\
& \left.\times \sum_{n=1}^{N_{F}}\left(\left\{\max \left(\mu_{n}^{2}(x)\right)\right\}^{2}\left\{\max \left\|\frac{\partial L_{n}}{\partial P_{n}^{l}(k)}\right\|\right\}^{2}\right)\right] .
\end{aligned}
$$

Thus, according to the Lyapunov stability theory, when $\lambda>0, \Delta V(k)<0$; that it to say, the output of the system can gradually track command signal and stability.

\section{Simulation Result and Analysis}

Because the moment of inertia will change greatly under different loading conditions and a large load variation and impact will occur in the rotating part when the weapon is 


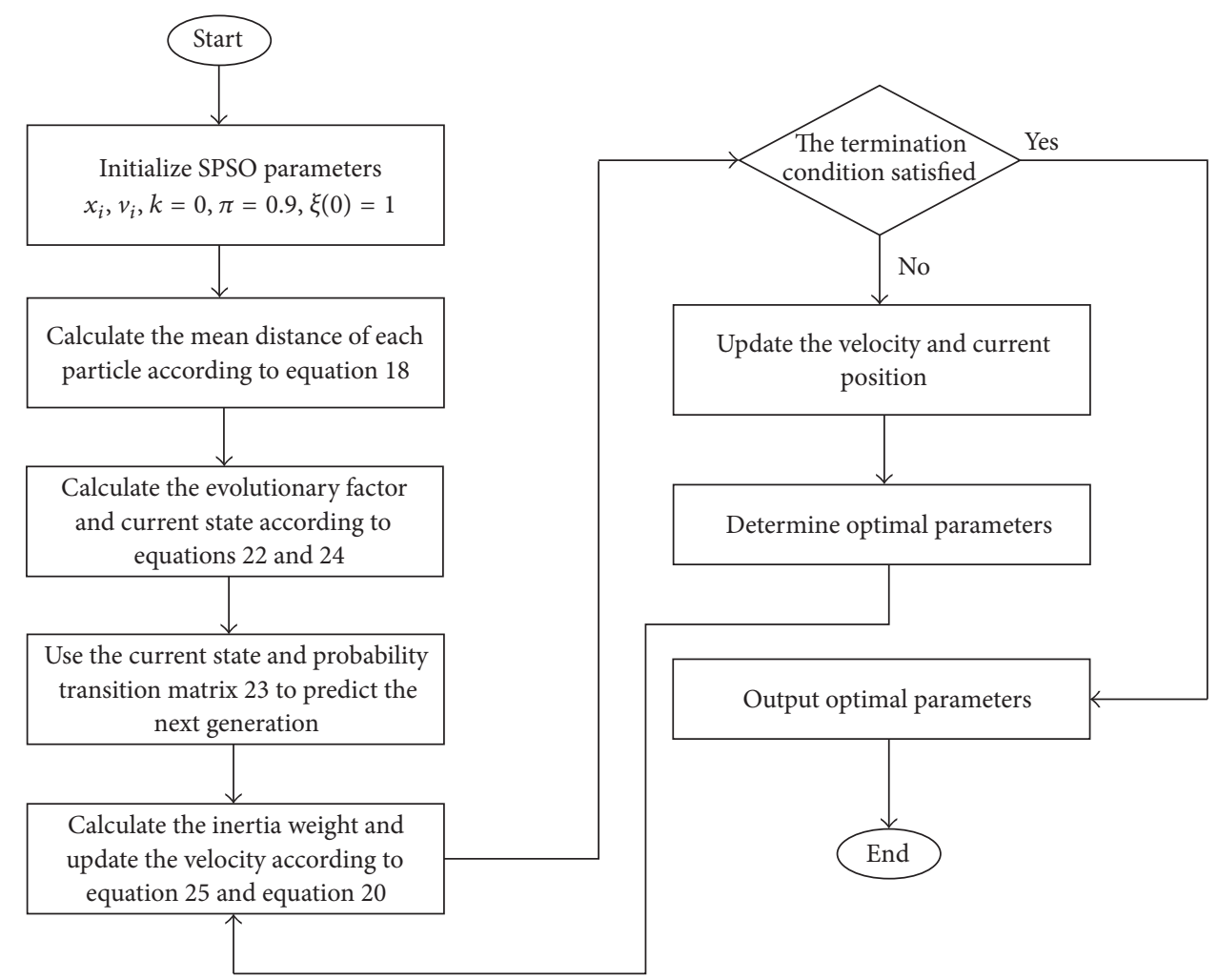

FIGURE 4: Flowchart of using the SPSO algorithm to optimize the parameters of WFNN.

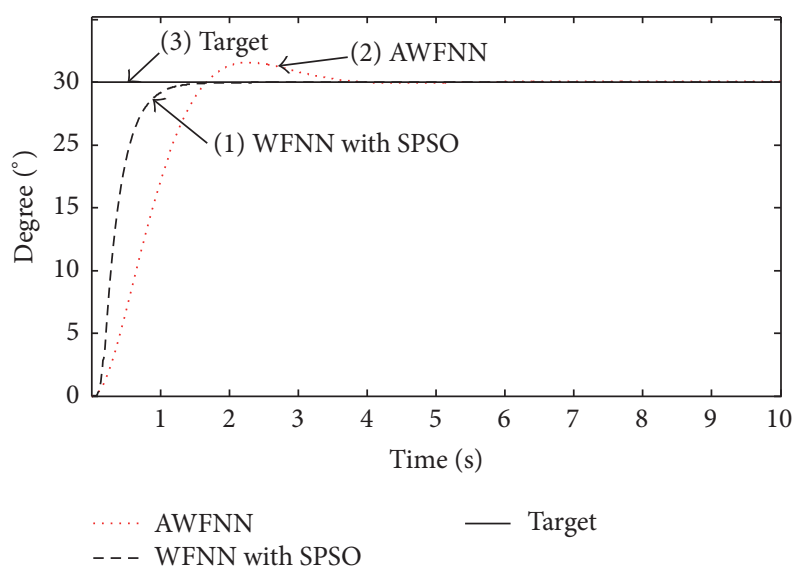

Figure 5: The moment of inertia changes step response curve.

launched, that make the traditional control method difficult to precise control system. Thus, a WFNN is applied to build the intelligent model and control for this system, which is introduced by previous section; moreover, adaptive parameters are trained by means of learning rate with using SPSO algorithm.

In order to verify the performance of the proposed fuzzy wavelet neural network controller, the adaptive fuzzy wavelet neural network (AWFNN) mode controller is compared. The simulation results are shown in Figures 5-9. In the figures, the black line 3 is the target output and black dotted line 1

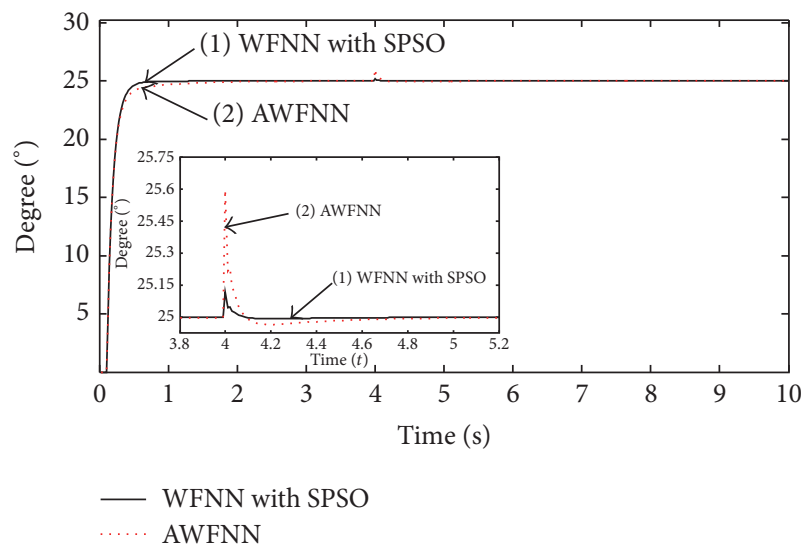

FIGURE 6: Step response curve of load disturbance.

is a wavelet fuzzy neural network with SPSO; and adaptive fuzzy wavelet neural network is shown by red dotted line 2. The main parameters of AC servo system are shown in Table 1. According to the dilation parameters of the select wavelets, five rules/sub-WNN are chosen for constructing each network. According to dilation parameters of selected wavelets, the proposed controller which is used contains 10 fuzzy rules with 10 membership functions which are assigned for each input variable.

Figure 5 for the moment of inertia is a variation of the initial value to 2 times the position of the response curve. When the moment of inertia changes, using adaptive wavelet 


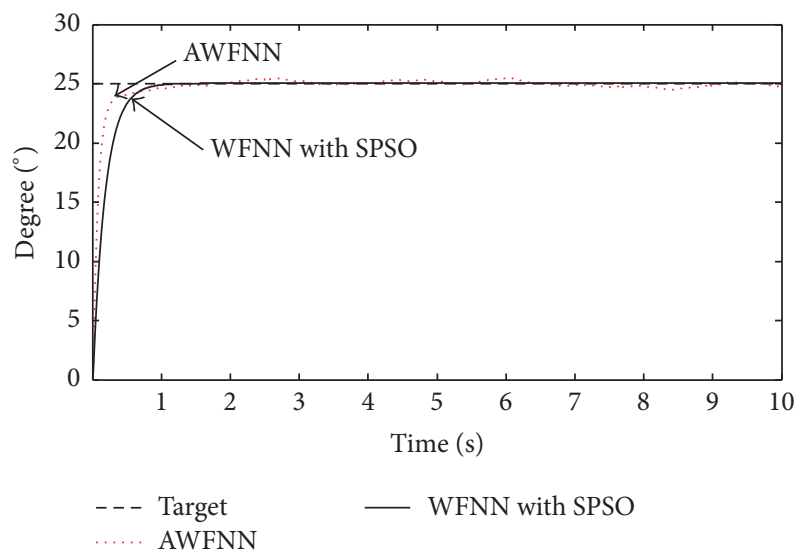

Figure 7: System dynamic response curves.

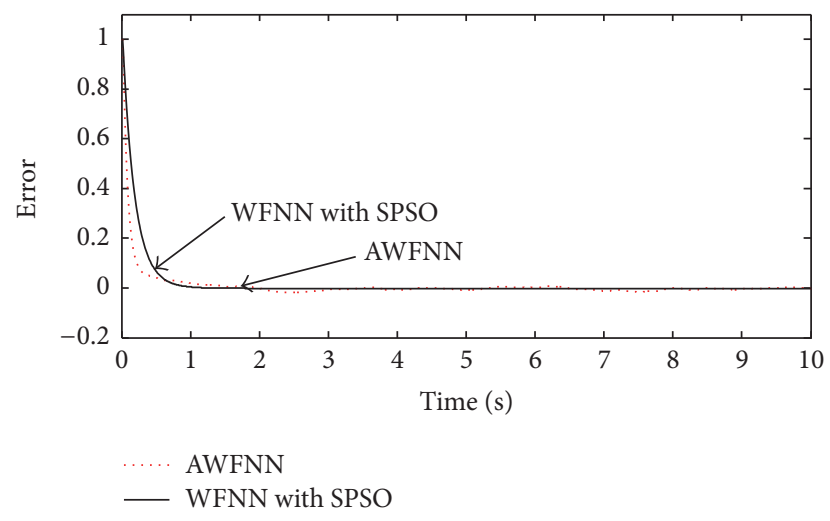

FIGURE 8: Error tracking system dynamic response curves.

TABLE 1: The main parameters of AC servo system.

\begin{tabular}{lccc}
\hline System parameters & $\mathrm{S}$ & Value & Unit \\
\hline $\begin{array}{l}\text { Load converted to the motor } \\
\text { output shaft moment of inertia }\end{array}$ & $J$ & 5239 & $\mathrm{Kg} \cdot \mathrm{m}^{2}$ \\
$\begin{array}{l}\text { Converted to unbalanced torque } \\
\text { and friction torque of the motor } \\
\text { output shaft }\end{array}$ & $T_{L}$ & $9.32 \times 10^{3}$ & $\mathrm{~N} \cdot \mathrm{m}$ \\
$\begin{array}{l}\text { Electromagnetic torque } \\
\text { coefficient }\end{array}$ & $K_{t}$ & 0.195 & $\mathrm{~N} \cdot \mathrm{m} / \mathrm{A}$ \\
$\begin{array}{l}\text { Friction moment of the load } \\
\text { Viscous friction coefficient }\end{array}$ & $T_{f}$ & 850 & $\mathrm{~kg} \cdot \mathrm{m}^{2}$ \\
Reduction ratio & $B$ & $1.43 \times 10^{-4}$ & $\mathrm{~N} \cdot \mathrm{m} /\left(\mathrm{rad} \cdot \mathrm{s}^{-1}\right)$ \\
\hline
\end{tabular}

fuzzy control, the system generates overshoot and needs $2.58 \mathrm{~s}$ to reach the target. But, using wavelet fuzzy neural network with SPSO control, the system reaches a steady state and only needs $1.67 \mathrm{~s}$ and no overshoot generated. Thus, by contrast it shows that using wavelet fuzzy neural network with SPSO control mode can better suppress the perturbation changes.

Figure 6 shows the position response curve added with a $450 \mathrm{Nm}$ step disturbance at $4 \mathrm{~s}$. As can be seen from the figure, when the load disturbance occurs, the location of the response using the algorithm of AWFNN control shows large deviation, and it needs $0.82 \mathrm{~s}$ to recover the

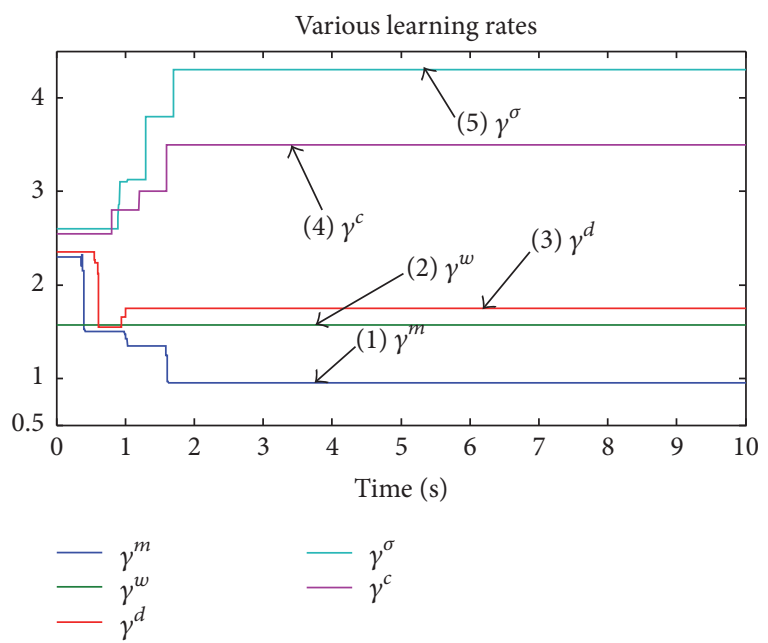

FIgURE 9: Various learning rates.

reference position; however, using WFNN with SPSO control algorithm, the system has better suppression performance of load disturbance, and the system offset is 0.035 degrees, and it only needs 0.1 second to reach the target position.

Figure 7 is a dynamic system to track the location of random interference, when added to the response curve; it can be seen that, when using AWFNN control, the system has been shifted up and down at the target position; however, using WFNN with SPSO control, the system did not produce offset. Thus, the WFNN with SPSO method has a strong antiinterference ability and good dynamic performance. Figure 8 is a dynamic system step response curve tracking error. It shows that when the system parameters are uncertain and external disturbances appear, the WFNN with SPSO controller can enable the tracking servo system to rapidly reach the given position signals and suppress the system impact of various uncertainties by introducing more efficient robust control. In other words, the accuracy and robustness of the system are improved.

In addition, SPSO can be used to adapt the learning rates online, which can be observed obviously as shown in Figure 9, where, line 1 , line 2 , line 3 , line 4 , and line 5 represent $\gamma^{m} ; \gamma^{w}$; $\gamma^{d} ; \gamma^{\sigma}$; and $\gamma^{c}$, respectively.

\section{Semiphysical Simulation Test}

To investigate the efficiency of the proposed WFNN with SPSO control as a strategy in establishing AC servo system, a semiphysical simulation platform is constructed to simulate the working conditions of the servo control system. The test results were compared to verify the performance of the controller in this paper superiority. The semiphysical simulation test-bed structure diagram and object diagram are shown in Figures 10 and 11, respectively.

Based on the components shown in Figure 10, the platform consists of seven parts, including the control computer, the sensor system for measurement, the power amplifier $(\mathrm{PA})$, the precision reduction gearbox (PRG), the loading 


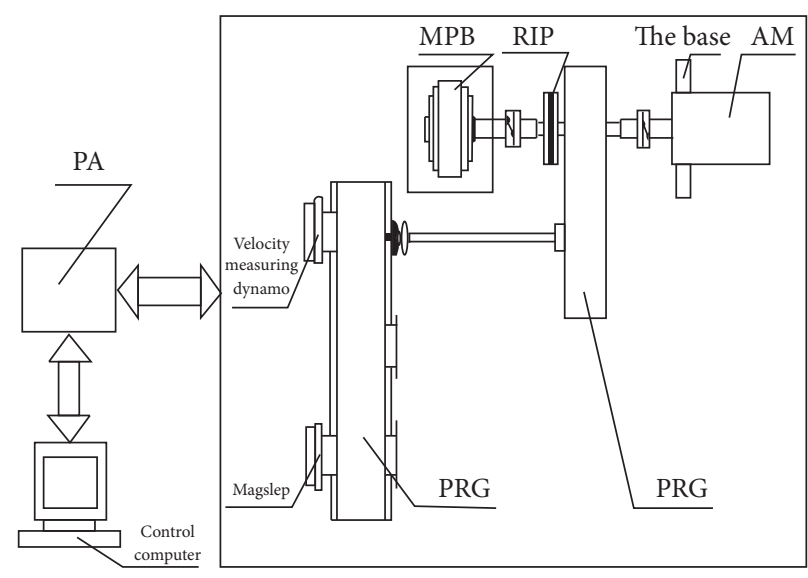

FIGURE 10: Schematic of the semiphysical simulation platform.
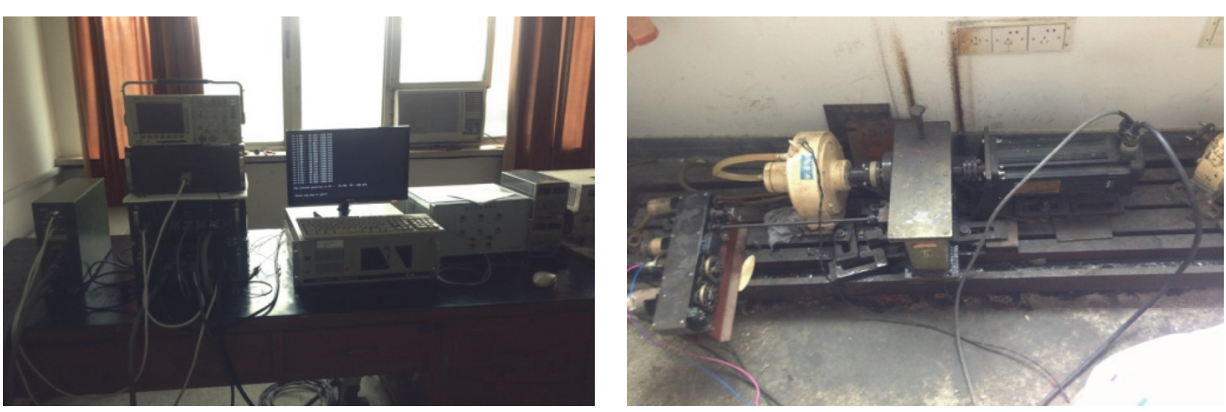

FIgURE 11: Photograph of the semiphysical simulation platform.

fixture (LF), the actuating motor (AM), and the test bed. The loading fixture, which consists of the rotational inertia plate (RIP) and the magnetic powder brake (MPB), is employed for the simulation of the rotational inertia, the load torque, and the frictional resistance moment. The rotational inertia variations in the loads are well simulated by changing the RIP. Similarly, the variations in the load torque and the frictional resistance moment are also well simulated by controlling the output torque of the MPB.

To investigate the tracking accuracy of the servo system with WFNN with SPSO control system, sinusoidal command tracking with an angle frequency of $16.7 \mathrm{rad} / \mathrm{s}$ and amplitude of 100 degree is conducted on the semiphysical simulation platform. The corresponding tracking curves of both the WFNN with SPSO and AWFNN control systems are illustrated in Figure 12.

By comparison, the WFNN with SPSO control system has a better dynamic performance and steady-state performance than the AWFNN control, and it can effectively increase the stability and speed of the system.

\section{Conclusion}

This study has demonstrated the validity of the proposed WFNN controller with SPSO for the position control of an AC servo system. First, the modeling of the AC servo

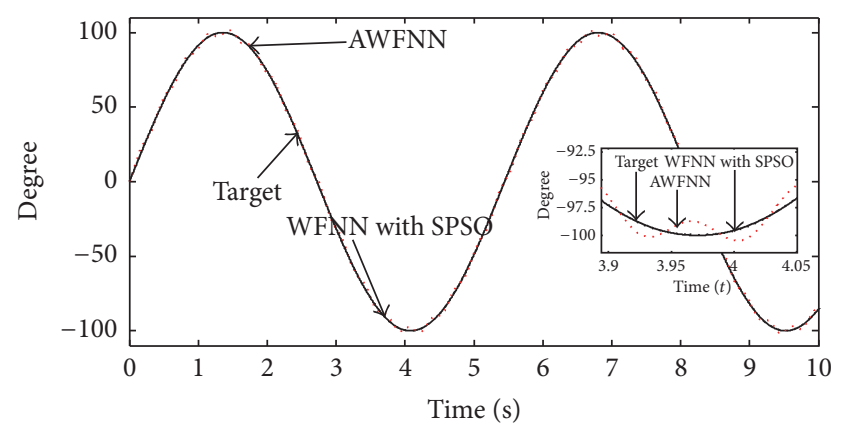

FIGURE 12: Sinusoidal tracking curves.

system was introduced. Then, the network structure and learning algorithm of the proposed WFNN controller were introduced in detail. Moreover, the SPSO algorithm has been adopted to adapt the learning rates online. Finally, the control performances of the AWFNN controller and proposed WFNN controller with SPSO have been compared by simulations and prototype test. From the simulation and prototype test results, the scheme of system uncertainties and external disturbance has strong robustness and good dynamic steady-state response performance. Therefore, the proposed WFNN controller with SPSO can control the AC servo system effectively. 


\section{Competing Interests}

The authors declare that they have no competing interests.

\section{References}

[1] M. R. Banaei and A. Kami, "Interline power flow controller (IPFC) based damping recurrent neural network controllers for enhancing stability," Energy Conversion and Management, vol. 52, no. 7, pp. 2629-2636, 2011.

[2] R.-C. Hwang, Y.-J. Chen, and H.-C. Huang, "Artificial intelligent analyzer for mechanical properties of rolled steel bar by using neural networks," Expert Systems with Applications, vol. 37, no. 4, pp. 3136-3139, 2010.

[3] Q. Gao, Y. Jin, Y.-L. Hou, and L.-J. Ji, "Modeling and control for mine sweeping plough electro-hydraulic servo system of certain mine-clearing vehicle," Journal of Nanjing University of Science and Technology, vol. 36, no. 2, pp. 238-244, 2012.

[4] Z. D. Qi, X. J. Zhu, and G. Y. Cao, "Neural networks modeling of MCFC system and fuzzy control research based on FGA," in Proceedings of the 5th World Congress on Intelligent Control and Automation (WCICA '04), pp. 2486-2490, Hangzhou, China, June 2004.

[5] Z. D. Qi, X. J. Zhu, and G. Y. Cao, "Voltage model of DMFC based on neural networks identification," Computer Simulation, vol. 22, no. 5, pp. 92-94, 2005.

[6] A. Sala, G. T. Marie, and R. Babuka, "Perspectives of fuzzy systems and control," Fuzzy Sets and Systems, vol. 156, no. 3, pp. 432-444, 2005.

[7] D.-W. Ding, X. Li, Y. Yin, and X.-P. Xie, "Further studies on relaxed stabilization conditions for discrete-time two-dimension Takagi-Sugeno fuzzy systems," Information Sciences, vol. 189, pp. 143-154, 2012.

[8] J. Dong, Y. Wang, and G.-H. Yang, "Output feedback fuzzy controller design with local nonlinear feedback laws for discretetime nonlinear systems," IEEE Transactions on Systems, Man, and Cybernetics, Part B: Cybernetics, vol. 40, no. 6, pp. 14471459, 2010.

[9] H. K. Lam and J. Lauber, "Membership-function-dependent stability analysis of fuzzy-model-based control systems using fuzzy Lyapunov functions," Information Sciences, vol. 232, pp. 253-266, 2013.

[10] J. Wang and J. Xiao, "Constructing fuzzy wavelet network modeling," in Proceedings of the International Conference on Intelligent Computing, pp. 169-172, Hefie, China, 2005.

[11] M. K. Kahkeshi, F. Sheikholeslam, and M. Zekri, "Design of adaptive fuzzy wavelet neural sliding mode controller for uncertain nonlinear systems," ISA Transactions, vol. 52, no. 3, pp. 342-350, 2013.

[12] R. H. Abiyev and O. Kaynak, "Fuzzy wavelet neural networks for identification and control of dynamic plants-a novel structure and a comparative study," IEEE Transactions on Industrial Electronics, vol. 55, no. 8, pp. 3133-3140, 2008.

[13] R. Cheng and Y. Bai, "A novel approach to fuzzy wavelet neural network modeling and optimization," International Journal of Electrical Power and Energy Systems, vol. 64, pp. 671-678, 2015.

[14] A. Ebadat, N. Noroozi, A. A. Safavi, and S. H. Mousavi, "New fuzzy wavelet network for modeling and control: the modeling approach," Communications in Nonlinear Science and Numerical Simulation, vol. 16, no. 8, pp. 3385-3396, 2011.
[15] M. Davanipoor, M. Zekri, and F. Sheikholeslam, "Fuzzy wavelet neural network with an accelerated hybrid learning algorithm," IEEE Transactions on Fuzzy Systems, vol. 20, no. 3, pp. 463-470, 2012.

[16] J. B. Oliveira, J. Boaventura Cunha, and P. B. Moura Oliveira, "A swarm intelligence-based tuning method for the sliding mode generalized predictive control," ISA Transactions, vol. 53, pp. 1501-1515, 2014.

[17] Y. Tang, Z. Wang, and J.-A. Fang, "Parameters identification of unknown delayed genetic regulatory networks by a switching particle swarm optimization algorithm," Expert Systems with Applications, vol. 38, no. 3, pp. 2523-2535, 2011.

[18] N. Zeng, Z. Wang, Y. Li, M. Du, and X. Liu, "A hybrid EKF and switching PSO algorithm for joint state and parameter estimation of lateral flow immunoassay models," IEEE/ACM Transactions on Computational Biology and Bioinformatics, vol. 9, no. 2, pp. 321-329, 2012.

[19] Q. Gao, Z. Sun, G. Yang, R. Hou, L. Wang, and Y. Hou, "A novel active disturbance rejection-based control strategy for a gun control system," Journal of Mechanical Science and Technology, vol. 26, no. 12, pp. 4141-4148, 2012.

[20] Y.-C. Zhu, D.-W. Ma, Z.-G. Li, and G.-G. Le, "Optimal sliding mode servo control with integral term for rocket launcher," Acta Armamentarii, vol. 28, no. 10, pp. 1272-1275, 2007.

[21] R. M. Hou, R. Z. Liu, Y. L. Hou, and Q. Gao, "High-power AC servo system identification research based on wavelet neural network," Applied Mechanics and Materials, vol. 220-223, pp. 997-1002, 2012.

[22] S. Ganjefar and M. Alizadeh, "Inter-area oscillations damping by multi-objective wavelet neural inverse controlled SSSC," International Review of Electrical Engineering, vol. 7, no. 2, pp. 4000-4012, 2012.

[23] S. Yilmaz and Y. Oysal, "Fuzzy wavelet neural network models for prediction and identification of dynamical systems," IEEE Transactions on Neural Networks, vol. 21, no. 10, pp. 1599-1609, 2010.

[24] Y. Tang, J.-A. Fang, and Q. Miao, "On the exponential synchronization of stochastic jumping chaotic neural networks with mixed delays and sector-bounded non-linearities," Neurocomputing, vol. 72, no. 7-9, pp. 1694-1701, 2009. 


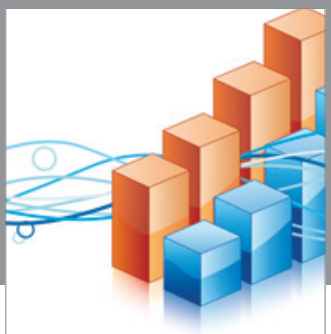

Advances in

Operations Research

vatem alat4

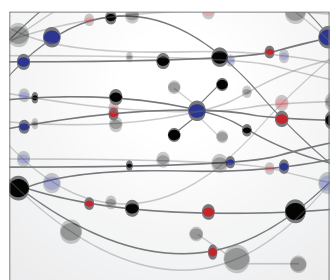

\section{The Scientific} World Journal
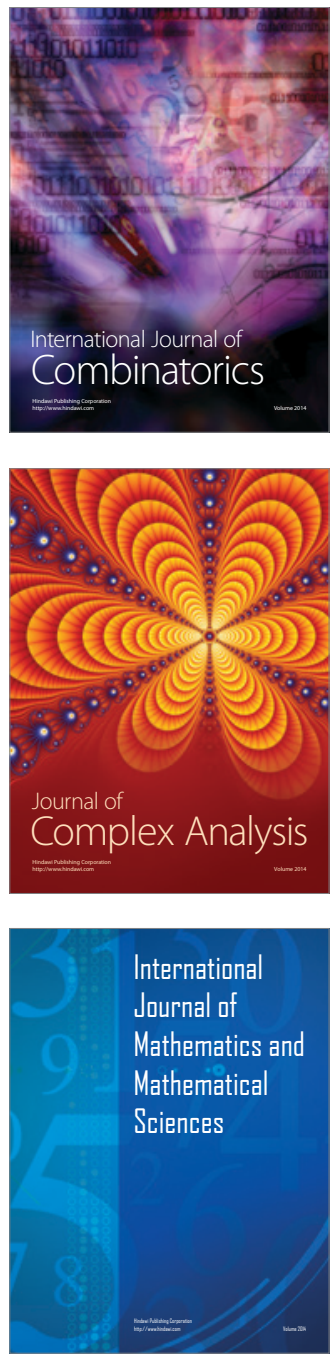
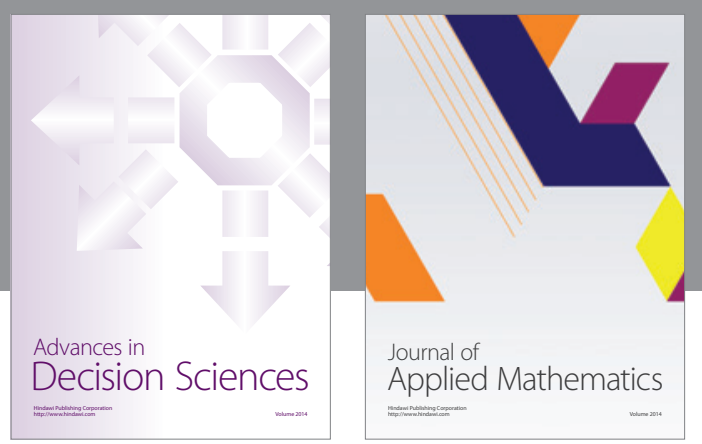

Algebra

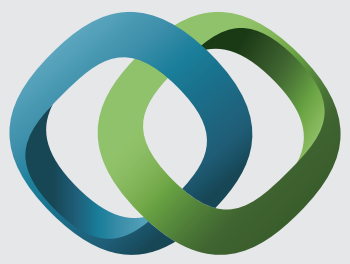

\section{Hindawi}

Submit your manuscripts at

http://www.hindawi.com
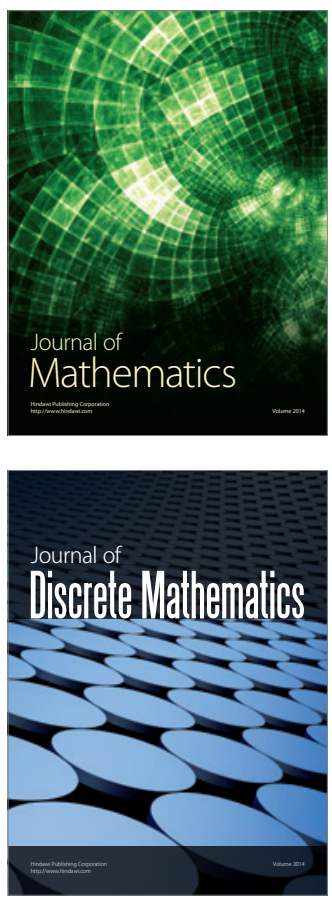

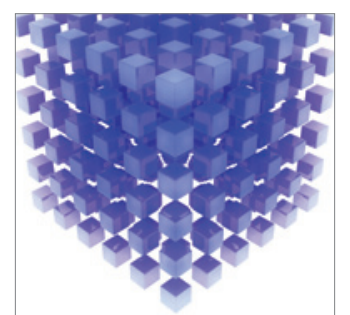

Mathematical Problems in Engineering
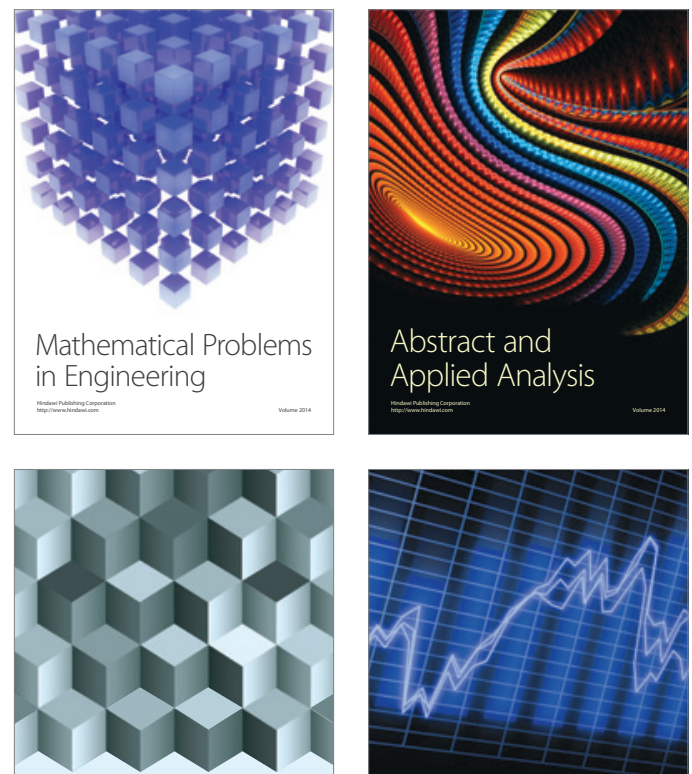

Journal of

Function Spaces

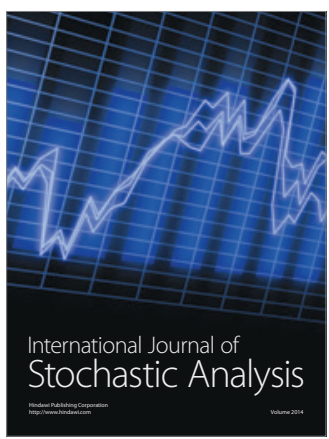

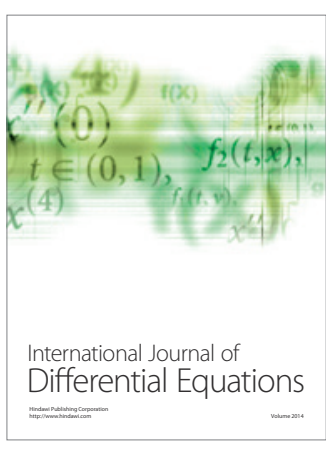
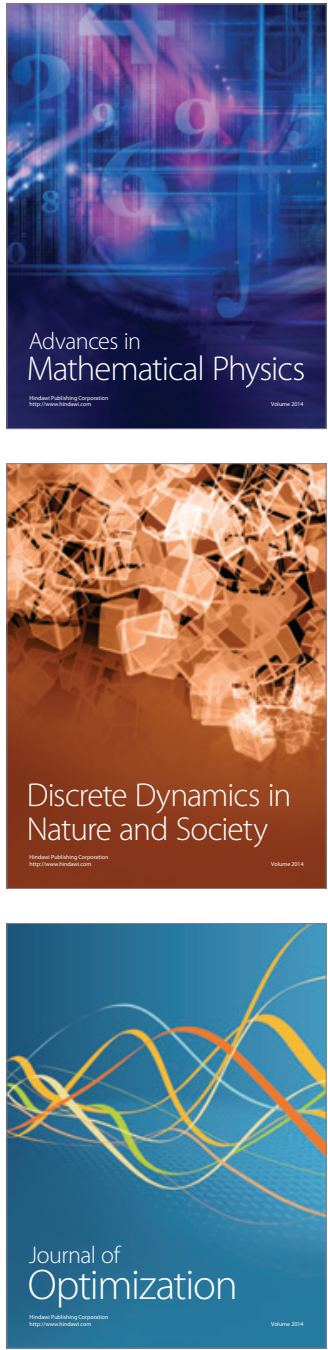